\title{
What have we learned about the processes involved in the lowa Gambling Task from developmental studies?
}

\author{
Mathieu Cassotti ${ }^{1,2 *}$, Ania Aïte ${ }^{1}$, Anaïs Osmont ${ }^{1}$, Olivier Houdé ${ }^{1,2}$ and Grégoire Borst ${ }^{1}$ \\ 1 Laboratory for the Psychology of Child Development and Education, CNRS Unit 8240, Sorbonne Paris Cité, Paris Descartes University, Paris, France \\ 2 Institut Universitaire de France, Paris, France
}

\author{
Edited by: \\ Ching-Hung Lin, Kaohsiung Medical \\ University, Taiwan \\ Reviewed by: \\ William Hedgcock, University of \\ lowa, USA \\ Maggie E. Toplak, York University, \\ Canada \\ *Correspondence: \\ Mathieu Cassotti, Laboratory for the \\ Psychology of Child Development \\ and Education, CNRS Unit 8240, \\ Université Paris Descartes, \\ Sorbonne, Laboratoire A. Binet, \\ 46 rue Saint Jacques, 75005 Paris, \\ France \\ e-mail:mathieu.cassotti@ \\ parisdescartes.fr
}

Developmental studies using the lowa Gambling Task (IGT) or child-friendly adaptations of the IGT converged in showing that children and adolescents exhibit a strong bias in favor of disadvantageous choices whereas adults learn to decide advantageously during the course of the task. In the present article, we reviewed developmental studies that used the IGT or child-friendly adaptations of the IGT to show how these findings provide a better understanding of the processes involved in decision-making under uncertainty. For instance, developmental studies have underlined that until late adolescence, the dominant strategy is to focus only on the frequency of punishment and to choose among options with infrequent losses. Indeed, school-aged children and adolescents' choices in the IGT seem to be guided by the loss frequency leading them to fail in distinguishing between advantageous and disadvantageous options. In addition, recent developmental studies revealed that adults switch less often after losses than school-aged children and adolescents. These findings suggest that psychological tolerance to loss may facilitate learning the characteristics of each option, which in turn improves the ability to choose advantageously. In conclusion, developmental studies help us refine our understanding of decision-making.

Keywords: lowa Gambling Task, emotion-based learning, executive control, inhibition (psychology), children and adolescents, developmental psychology, loss aversion

\section{INTRODUCTION}

In most situations of everyday life, people make decisions in circumstances where some information about the potential outcomes of choices are lacking and must be inferred from experience. Over the last two decades, considerable efforts in the field of psychology and neuroscience have been leveled at identifying the processes involved in this category of decision-making situations (Bechara and Damasio, 2005; Dunn et al., 2006). In particular, from a developmental perspective, a growing body of research suggests that the ability to decide advantageously evolves with age (see Table 1; Crone et al., 2005; Cassotti et al., 2011; Aite et al., 2012; Beitz et al., 2014). Therefore, in the present article, we reviewed these developmental studies to demonstrate how it helps us refine our understanding of decision-making under uncertainty.

According to the Somatic Marker Hypothesis (SMH) (Bechara et al., 2000; Bechara and Damasio, 2005), emotion-related signals, developed from past experience of the emotional consequences following choices, guide decision making in situations of uncertainty. More specifically, this theory assumes that advantageous decisions rely on the development of an integral emotional reactivity (i.e., somatic maker). These somatic markers allow one to avoid disadvantageous options and to develop a preference for advantageous ones.

Most of the empirical supports for this model came from studies using the Iowa Gambling Task (IGT, Bechara and Damasio,
2005), a task initially developed to simulate the inherent uncertainty of daily-life decisions' situations through an opaque gain-loss schedule. The IGT consists of a card game in which participants are instructed to win as much money as possible by selecting among four possible decks of cards (labeled A, B, C, or D). Importantly, the decks' characteristics are not disclosed, and should be gradually inferred from feedbacks obtained during the game. Indeed, feedbacks are provided after each selection so that participants systematically win some money, but also and unforeseeably lose some. The four decks differ in the magnitude of wins and losses in such a way that to succeed at this game, players must withdraw from attractive but disadvantageous in the long term decks (A, B) and opt for less attractive but advantageous in the long term decks (C, D).

Typically, healthy adults progressively and implicitly learn to choose advantageously during the course of the task (Crone and van der Molen, 2004; Dunn et al., 2006; Cassotti and Moutier, 2010; Cassotti et al., 2011; Turnbull et al., 2014). In addition, advantageous performances has been linked to an anticipated emotional reactivity, as measured by Skin Conductance Responses (SCRs). Indeed, healthy participants display gradually higher anticipatory SCRs before picking a card in disadvantageous decks than in advantageous ones, suggesting that an emotional warning signal leads them to avoid disadvantageous choices (Bechara et al., 2000; Carter and Smith-Pasqualini, 2004; Guillaume et al., 2009). Similarly, anticipatory heart rate 


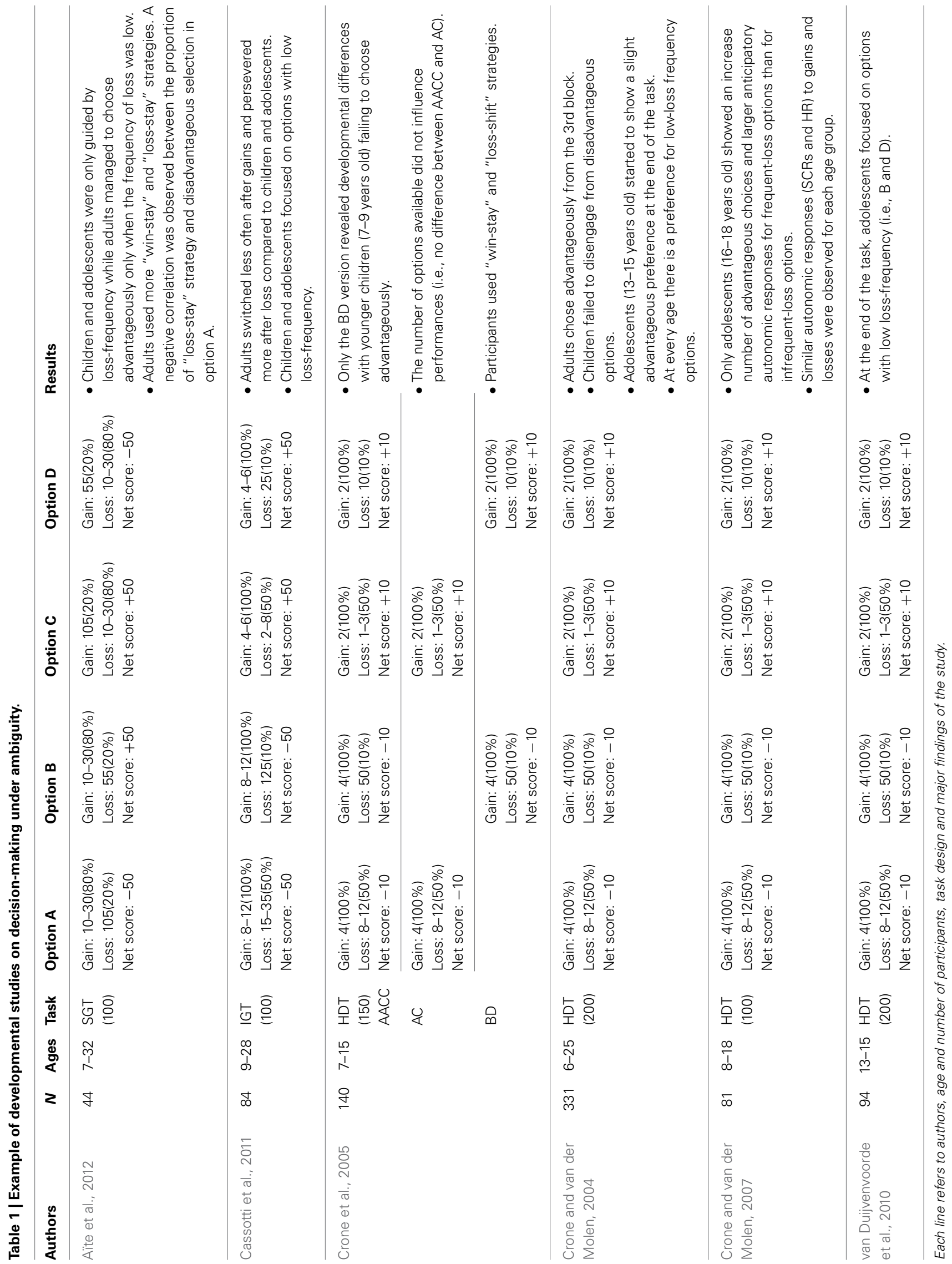


responses and SCRs were found to be critical to distinguish good and bad performers in such decision-making under uncertainty tasks suggesting a key role of this anticipated emotional reactivity in the ability to make advantageous decisions (Crone et al., 2004; Denburg et al., 2006).

In agreement with the SMH both neuropsychological studies and neuroimaging studies have shown the involvement of specific brain regions implicated in emotional processes in the IGT (Reimann and Bechara, 2010). More specifically, poor IGT performance and lower anticipatory SCRs were observed in amygdala-damaged patients (Bechara et al., 1999) as well as in patients with ventromedial prefrontal cortex (VMPFC) lesions (Bechara and Damasio, 2005). Using functional Magnetic Resonance Imaging, several studies have reported the activation of a prefrontal network including the VMPFC in the IGT which provides convergent evidence that decision-making under uncertainty might rely on an emotional neural circuitry (Lawrence et al., 2008; Li et al., 2010).

Additional empirical evidences in favor of the $\mathrm{SMH}$ came from studies exploring the influence of the emotional context on advantageous decisions in the IGT (Hinson et al., 2006; Davies and Turnbull, 2011; Aïte et al., 2013; see Turnbull et al., 2014 for a review). In response to remaining critics about the reliability of electrophysiological measures such as SCRs (Tomb et al., 2002; Dunn et al., 2006), Aite et al. (2013) recently designed an emotional priming paradigm of the IGT to determine whether the ability to choose advantageously in ambiguous situations is driven by an integral emotional signal as assumed by the SMH. In this study, the emotional context was either congruent or incongruent with the feedback delivered after each choice and was manipulated using pictures of either happy or fearful faces (Tottenham et al., 2009). The results of this study strongly support the SMH by evidencing that decision making was improved when the integral emotional signal was reinforced by a congruent emotional context and impaired when the integral emotional signal was disrupted by an incongruent emotional context.

\section{CHILD FRIENDLY ADAPTATION OF THE IGT AND ADVANTAGEOUS DECISION-MAKING}

Given that neuroimaging studies over the past 20 years have consistently shown continuing neuroanatomical and neurofunctional development of the prefrontal cortex across childhood and adolescence (Crone and Dahl, 2012), numerous developmental studies examining decision making under uncertainty have focused on changes in performance on child friendly adaptations of the IGT between school-aged children and adolescence (Crone et al., 2005; Cassotti et al., 2011; Beitz et al., 2014).

One of the first studies that investigated developmental changes in decision-making ability during adolescence showed that this ability continues to improve until late adolescence and even in adulthood (Crone and van der Molen, 2004). The authors of this study have designed an age-appropriate version of the IGT: the Hungry Donkey Task (HDT), in which the stimulus presentation was modified to make the task more meaningful for children (Crone and van der Molen, 2004). Indeed, rather than picking cards to win money for themselves, participants are invited to assist a hungry donkey in winning as many apples as possible by opening doors. Two doors (A and B) constitute disadvantageous choices (resulting in overall loss), and two doors ( $\mathrm{C}$ and D) advantageous choices (resulting in overall gain). Critically, this task maintains the basic format and a similar schedule of rewards and losses as those described by Bechara et al. (1994). As in other IGT studies, participants' performance is measured in terms of changes in individuals' net scores for blocks of 20 trials by subtracting the number of choices in disadvantageous doors from the number choices in advantageous doors.

In a series of behavioral studies, Crone and colleagues have demonstrated that 6- to 9-year-old children and 10- to 12-yearold children fail to avoid disadvantageous options during the course of the task, as opposed to 13- to 15 year-old adolescents, who gradually learn to choose advantageously (Crone and van der Molen, 2004, 2007; Crone et al., 2005; Huizenga et al., 2007). Nevertheless, adolescents' performance is still suboptimal compared to adults, suggesting that the ability to distinguish between advantageous and disadvantageous options continues to improve during late adolescence (Overman, 2004; Overman and Pierce, 2013). Although some authors point out that there may be differences between the development of decision making involving personal gain or loss and decision making that leads to help another such as in the HDT, developmental studies using the standard IGT have confirmed that advantageous decision making progressively increases until early adulthood (Hooper et al., 2004; Cassotti et al., 2011; Beitz et al., 2014). It has been proposed that this age-related improvement in performance on child adaptations of the IGT during adolescence may be due the slow functional maturation of the VMPFC until early adulthood (Crone and van der Molen, 2004, 2007).

\section{DEVELOPMENT OF THE FREOUENCY BIAS}

Because the IGT is a complex task, different factors may contribute to the similar decision-making deficit observed in children and VMPFC patients. In support of this hypothesis, developmental studies revealed that children have a marked preference for options associated with infrequent losses, regardless of whether these options are advantageous or disadvantageous in the long run (Crone and van der Molen, 2004, 2007; Crone et al., 2005; Huizenga et al., 2007; Carlson et al., 2009; Cassotti et al., 2011). Indeed, the four options proposed in the IGT and the childfriendly versions of this task also differ in the frequency of losses, with two options associated with a low frequency of losses $(10 \%$ for decks/doors B and D) and two options associated with a medium frequency of losses (50\% for decks/doors A and C). Developmental studies converged in showing that children and adolescents increasingly opt for choices associated with infrequent, rather than frequent losses during the task and that this frequency bias decreases with age (Huizenga et al., 2007). In contrast, adults display a strong preference for low loss frequency choices early in the task but progressively opt for advantageous options during the course of the task. To the best of our knowledge, this specific sensitivity to options associated with a low frequency of losses has not been observed in VMPFC patients.

Furthermore, Crone and van der Molen (2007) demonstrated that adolescents display higher anticipatory SCRs preceding a choice among options associated with frequent losses in contrast 
to adults who generate progressively higher anticipatory SCRs before disadvantageous selections (Bechara and Damasio, 2005). These results suggest (a) that loss frequency highly influences children and adolescents' decision making, and (b) that children and adolescents exhibit difficulties in considering both the frequency and the amount of loss leading them to make long-term disadvantageous decisions (see also Jansen et al., 2011; van Duijvenvoorde et al., 2012). Interpreted within the framework of the SMH, these data suggest that adolescents reactivate the negative emotional responses associated with high-loss frequency options without taking into account the final outcome.

Interestingly, recent evidence underlined a comparable inability to integrate both loss frequency and final outcome in adults' decision making (Lin et al., 2009; Cassotti and Moutier, 2010). For example, using the Soochow Gambling Task (SGT), an adaptation of the IGT designed to directly contrast the impact of loss frequency and final outcome on decision making, Lin et al. (2009) demonstrated that adults substantially based their choices on loss frequency rather than on final outcome (i.e., the advantageous or disadvantageous nature of the decks on the long run). More specifically, when the frequency of loss in the decks is manipulated to take either one of two extreme values ( 80 vs. $20 \%$ ), adults are guided by gain-loss frequency leading them to fail in distinguishing between advantageous and disadvantageous choices. Given that this loss-frequency bias was observed only in children and adolescents when the classical IGT was used, Aite et al. (2012) further explored developmental changes in the ability to consider both the loss frequency and the final outcome in decision making using an age adapted version of the SGT. Results confirmed that children and adolescent not only preferred choices associated with infrequent losses in the SGT but also failed to differentiate advantageous options from disadvantageous ones, a developmental pattern similar to the one previously evidenced using the IGT. Contrarily to Lin et al.'s study (2009), findings indicated that adults did manage to consider the final outcome when making their decision but only for options associated with low-loss frequency. Thus, adults are not only guided by the loss frequency but also by the amount of the loss as evidenced by a preference for the advantageous option among the low-loss frequency options. Taken together, developmental studies using the IGT, the HDT, or the SGT converge in showing a robust frequency bias in childhood and adolescent that decrease in adults (Crone et al., 2005; Crone and van der Molen, 2007; Aïte et al., 2012).

\section{STRATEGIC ADJUSTMENTS OF DECISION MAKING}

A large majority of previous studies measured performance in the IGT or child adaptations of the IGT in terms of changes in difference between the number of advantageous selections and the number of disadvantageous choices. However, such measures provide no information on the strategic adjustments that immediately follow gains and losses over the course of the task. Given that participants can chose any of the four options on each trial, age differences observed in the standard net score and the tendency to focus on loss frequency could result from age differences in the strategic exploration of the four options.

In line with this hypothesis, Cassotti et al. (2011) have recently examined response-switching behavior following rewards and punishments in children, adolescents and adults. In this study, adults tended to persevere with the same choice after a win (i.e., a "win-stay" strategy) and to shift to a new choice after a loss (i.e., a "loss-shift" strategy). In contrast, children and adolescents failed to control a spontaneous tendency to explore the different options as shown by a higher frequency of switches following gains and losses in children and adolescents than in adults. Another study has not only confirmed these developmental differences but has also outlined a possible role for these strategic adjustments following gains and losses (Aïte et al., 2012). Given that the proportion of switches after losses correlated negatively with the number of advantageous selections, the reduced lossshift pattern of response observed in adults, as compared to the two younger groups, could constitute a critical adaptive process allowing one to choose advantageously. Indeed, adults' tolerance to loss may allow them to learn the characteristics of each option and to increase their ability to consider not only loss frequency but also final outcome.

In line with these developmental studies (Cassotti et al., 2011; Aite et al., 2012; van Duijvenvoorde et al., 2012) computational models that have included win-stay/loss-shift strategies to predict performance in the IGT (Worthy et al., 2007; Worthy and Maddox, 2012) converged in showing that the tendency to inhibit the loss-shift response is a central component of decision-making behavior in the IGT. Altogether, these results suggest that immature decision making may be due to the difficulty to execute inhibitory control on an automatic loss-shift response.

\section{CONCLUSION}

In the present review, we have discussed how developmental studies have made significant progress in the understanding of the processes involved in emotional-based learning in the IGT. Behavioral and electrophysiological studies have clearly demonstrated a focus on loss-frequency (Crone et al., 2005; Crone and van der Molen, 2007) and a preponderance of a loss-shift strategy in children and adolescent as compared to adults (Aïte et al., 2012). In line with recent behavioral and neuroimaging studies (Spear, 2011; Habib et al., in press) children and adolescent might be more focused on loss frequency and might rely more on a loss-shift strategy because of an exacerbate aversion to losses. Given that only adults have the ability to choose advantageously (Crone and van der Molen, 2004, 2007; Overman, 2004; Overman and Pierce, 2013), the subtle developmental differences regarding the factors that guide decision making and the strategic adjustment that are used might reflect the critical components needed for making advantageous decisions. Indeed, the data collected in developmental studies suggest that the ability to develop emotion-related signals that integrate both loss-frequency and final outcome requires inhibitory control of intuitive exploration strategies. As such, the present article provides new fuel for the current debates on the respective contribution of executive control and emotion-based learning in the IGT.

\section{REFERENCES}

Aïte, A., Borst, G., Moutier, S., Varescon, I., Brown, I., Houdé, O., et al. (2013). Impact of emotional context congruency on decision making under ambiguity. Emotion 13, 177-182. doi: 10.1037/a0031345 
Aïte, A., Cassotti, M., Rossi, S., Poirel, N., Lubin, A., Houdé, O., et al. (2012). Is human decision making under ambiguity guided by loss frequency regardless of the costs? A developmental study using the Soochow Gambling Task. J. Exp. Child Psychol. 113, 286-294. doi: 10.1016/j.jecp.2012.05.008

Bechara, A., and Damasio, A. (2005). The somatic marker hypothesis: a neural theory of economic decision. Games Econ. Behav. 52, 336-372. doi: 10.1016/j.geb.2004.06.010

Bechara, A., Damasio, A. R., Damasio, H., and Anderson, S. W. (1994). Insensitivity to future consequences following damage to human prefrontal cortex. Cognition 50, 7-15. doi: 10.1016/0010-0277(94)90018-3

Bechara, A., Damasio, H., Damasio, A., and Lee, G. (1999). Different contributions of the human amygdala and ventromedial prefrontal cortex to decision-making. J. Neurosci. 19, 5473-5481.

Bechara, A., Damasio, H., and Damasio, A. R. (2000). Emotion, decision-making, and the orbitofrontal cortex. Cereb. Cortex 10, 295-307. doi: 10.1093/cercor/10.3.295

Beitz, K. M., Salthouse, T. A., and Davis, H. P. (2014). Performance on the Iowa Gambling Task: from 5 to 89 years of age. J. Exp. Psychol. Gen. 143, 1677-1689. doi: $10.1037 / \mathrm{a} 0035823$

Carlson, S. M., Zayas, V., and Guthormsen, A. (2009). Neural correlates of decision making on a Gambling Task. Child Dev. 80, 1076-1096. doi: 10.1111/j.14678624.2009.01318.x

Carter, S., and Smith-Pasqualini, M. C. (2004). Stronger autonomic response accompanies better learning: a test of Damasio's somatic marker hypothesis Cogn. Emot. 18, 901-911. doi: 10.1080/02699930341000338

Cassotti, M., Houdé, O., and Moutier, S. (2011). Developmental changes of win-stay and loss-shift strategies in decision making. Child Neuropsychol. 17, 400-411. doi: 10.1080/09297049.2010.547463

Cassotti, M., and Moutier, S. (2010). How to explain receptivity to conjunctionfallacy inhibition training: evidence from the Iowa Gambling Task. Brain Cognit. 73, 378-384. doi: 10.1016/j.bandc.2009.11.004

Crone, E., and van der Molen, M. W. (2007). Development of decision making in school-aged children and adolescents: evidence from heart rate and skin conductance analysis. Child Dev. 78, 1288-1301. doi: 10.1111/j.14678624.2007.01066.x

Crone, E. A., Bunge, S. A., Latenstein, H., and van der Molen, M. W. (2005). Characterization of children's decision making: sensitivity to punishment frequency, not task complexity. Child Neuropsychol. 11, 245-263. doi: 10.1080/092970490911261

Crone, E. A., and Dahl, R. E. (2012). Understanding adolescence as a period of social-affective engagement and goal flexibility. Nat. Rev. Neurosci. 13, 636-650. doi: $10.1038 / \mathrm{nrn} 3313$

Crone, E. A., Somsen, R. J., Beek, B. V., and van der Molen, M. W. (2004). Heart rate and skin conductance analysis of antecendents and consequences of decision making. Psychophysiology 41, 531-540. doi: 10.1111/j.1469-8986.2004.00197.x

Crone, E. A., and van der Molen, M. W. (2004). Developmental changes in decision-making: performance on a Gambling Task previously shown to rely on ventromedial prefrontal cortex. Dev. Neuropsychol. 25, 251-279. doi: $10.1207 / \mathrm{s} 15326942 \mathrm{dn} 2503 \_2$

Davies, J. L., and Turnbull, O. H. (2011). Affective bias in complex decision making: modulating sensitivity to aversive feedback. Motiv. Emot. 35, 235-248. doi: 10.1007/s11031-011-9217-x

Denburg, N. L., Recknor, E. C., Bechara, A., and Tranel, D. (2006). Psychophysiological anticipation of positive outcomes promotes advantageous decision-making in normal older persons. Int. J. Psychophysiol. 61, 19-25. doi: 10.1016/j.ijpsycho.2005.10.021

Dunn, B. D., Dalgleish, T., and Lawrence, A. D. (2006). The somatic marker hypothesis: a critical evaluation. Neurosci. Biobehav. Rev. 30, 1-33. doi 10.1016/j.neubiorev.2005.07.001

Guillaume, S., Jollant, F., Jaussent, I., Lawrence, N., Malafosse, A., and Courtet, P. (2009). Somatic markers and explicit knowledge are both involved in decisionmaking. Neuropsychologia 47, 2120-2124. doi: 10.1016/j.neuropsychologia. 2009.04.003

Habib, M., Borst, G., Poirel, N., Houdé, O., Moutier, S., and Cassotti, M. (in press). Socio-emotional context and adolescents' decision making: the experience of regret and relief after social comparison. J. Res. Adolesc. doi: 10.1111/jora. 12092

Hinson, J. M., Whitney, P., Holben, H., and Wirick, A. K. (2006). Affective biasing of choices in Gambling Task decision making. Cogn. Affect. Behav. Neurosci. 6, 190-200. doi: 10.3758/CABN.6.3.190
Hooper, C. J., Luciana, M., Conklin, H. M., and Yarger, R. S. (2004). Adolescents performance on the Iowa Gambling Task: implications for the development of decision making and ventromedial prefrontal cortex. Dev. Psychol. 40, 1148-1158. doi: 10.1037/0012-1649.40.6.1148

Huizenga, H. M., Crone, E. A., and Jansen, B. J. (2007). Decision-making in healthy children, adolescents and adults explained by the use of increasingly complex proportional reasoning rules. Dev. Sci. 10, 814-825. doi: 10.1111/j.14677687.2007.00621.x

Jansen, B. R. J., van Duijvenvoorde, A., and Huizenga, H. M. (2011). Development of decision-making: sequential versus integrative rules. J. Exp. Child Psychol. 111, 87-100. doi: 10.1016/j.jecp.2011.07.006

Lawrence, N. S., Jollant, F., O’Daly, O., Zelaya, F., and Phillips, M. L. (2008). Distinct roles of prefrontal cortical subregions in the Iowa Gambling Task. Cereb. Cortex 19, 1134-1143. doi: 10.1093/cercor/bhn154

Li, X., Lu, Z. L., D’Argembeau, A., Ng, M., and Bechara, A. (2010). The Iowa Gambling Task in fMRI images. Hum. Brain Mapp. 31, 410-423. doi: $10.1002 / \mathrm{hbm} .20875$

Lin, C. H., Chiu, Y. C., and Huang, T. H. (2009). Gain-loss frequency and final outcome in the Soochow Gambling Task: a reassessment. Behav. Brain Funct. 5:45. doi: 10.1186/1744-9081-5-45

Overman, W. H. (2004). Sex differences in early childhood, adolescence and adulthood on cognitive tasks that rely on orbital prefrontal cortex. Brain Cognit. 55, 134-147. doi: 10.1016/S0278-2626(03)00279-3

Overman, W. H., and Pierce, A. (2013). Iowa Gambling Task with non-clinical participants: effects of using real + virtual cards and additional trials. Front. Psychol 4:935. doi: 10.3389/fpsyg.2013.00935

Reimann, M., and Bechara, A. (2010). The somatic marker framework as a neurological theory of decision-making: review, conceptual comparisons, and future neuroeconomics research. J. Econ. Psychol. 31, 767-776. doi: 10.1016/j.joep.2010.03.002

Spear, L. P. (2011). Rewards, aversions, and affect in adolescence. emerging convergences across laboratory animal and human data. Dev. Cogn. Neurosci. 1, 390-403. doi: 10.1016/j.den.2011.08.001

Tomb, I., Hauser, M., Deldin, P., and Caramazza, A. (2002). Do somatic markers mediate decisions on the Gambling Task? Nat. Neurosci. 5, 1103-1104. doi: $10.1038 / \mathrm{nn} 1102-1103$

Tottenham, N., Tanaka, J. W., Leon, A. C., McCarry, T., Nurse, M., Hare, T. A., et al. (2009). The NimStim set of facial expressions: judgments from untrained research participants. Psychiatry Res. 168, 242-249. doi: 10.1016/j.psychres. 2008.05.006

Turnbull, O. H., Bowman, C. H., Shanker, S., and Davies, J. L. (2014). Emotionbased learning: insights from the Iowa Gambling Task. Front. Psychol. 5:162. doi: 10.3389/fpsyg.2014.00162

van Duijvenvoorde, A. C. K., Jansen, B. R. J., Bredman, J. C., and Huizenga, H. M. (2012). Age-related changes in decision making: comparing informed and non informed situations. Dev. Psychol. 48, 192-203. doi: 10.1037/a0025601

van Duijvenvoorde, A., Jansen, B. R. J., Visser, I., and Huizenga, H. M. (2010). Affective and cognitive decision making in adolescents. Dev. Neuropsychol. 35, 539-554. doi: 10.1080/87565641.2010.494749

Worthy, D. A., and Maddox, W. T. (2012). Age-based differences in strategy use in choice tasks. Front. Neurosci. 5:145. doi: 10.3389/fnins.2011.00145

Worthy, D. A., Maddox, W. T., and Markman, A. B. (2007). Regulatory fit effects in a choice task. Psychon. Bull. Rev. 14, 1125-1132. doi: 10.3758/BF03193101

Conflict of Interest Statement: The authors declare that the research was conducted in the absence of any commercial or financial relationships that could be construed as a potential conflict of interest.

Received: 30 March 2014; accepted: 31 July 2014; published online: 20 August 2014 Citation: Cassotti M, Aïte A, Osmont A, Houdé O and Borst G (2014) What have we learned about the processes involved in the Iowa Gambling Task from developmental studies? Front. Psychol. 5:915. doi: 10.3389/fpsyg.2014.00915

This article was submitted to Decision Neuroscience, a section of the journal Frontiers in Psychology.

Copyright (c) 2014 Cassotti, Aïte, Osmont, Houdé and Borst. This is an open-access article distributed under the terms of the Creative Commons Attribution License (CC BY). The use, distribution or reproduction in other forums is permitted, provided the original author(s) or licensor are credited and that the original publication in this journal is cited, in accordance with accepted academic practice. No use, distribution or reproduction is permitted which does not comply with these terms. 